\title{
PTGS2, CD133, CDK5 knockout comparison by CRISPR CAS-9 system in UV-Induced and Metastatic Melanoma
}

\author{
Rohan Priyam¹, Nicole Guilz² and Allison Cuppia ${ }^{1}$ \\ ${ }^{1}$ Mount Pisgah Christian School, Johns Creek, GA, USA \\ ${ }^{2}$ Columbia University, New York, NY, USA
}

\section{ABSTRACT}

CRISPR Cas9 systems have become an increasingly powerful method to express and repress gene expression. Within cancer research, CRISPR Cas9 technology can be regarded as a tool to knockout or knockin specific genes, ultimately causing metastasis. In this research, the knockout and knockin of the PTGS2, CD133, CDK5 genes are compared to determine whether a knockout or knockin of a specific gene can improve a metastatic melanoma patient's prognosis. Melanoma is a type of skin cancer with a stage IV survival rate of $22.5 \%$. Several melanoma patients have mutations in the PTGS2, CD133, CDK5 genes, causing metastasis. The knockdown of the PTGS2 gene reduces expression, which inhibits cell proliferation, migration, and invasiveness, modulates immune response by impairing myeloidderived suppressor cell differentiation, and reduces tumor development and metastasis in vivo. In comparison, the induced knockdown of the CD133 gene results in enhanced invasion and improved MMP2/MMP9 concentrations. MMP2/MMP9 concentrations encode for proteins that degrade type IV collagen, which considers the CD133 gene expression as a key role in tumor growth and metastasis. Lastly, the knockin of CDK5 may be a promising alternative as it controls melanoma cell motility, invasiveness, and metastatic spread.

\section{Introduction}

In recent years, UV-induced melanoma and Metastatic melanoma cases have drastically increased. In a study by Sandru A. et al, $80.6 \%$ of patients with metastatic melanoma died during the study. In addition, patients that survived the length of the conducted study, had a patient prognosis of a lowly 5.3 months (Sandru et al., 2014). Stage I melanoma is considered a local melanoma. Usually no evidence for metastasis to lymph nodes, lymph tissues, or body organs (Stage 1 Melanoma - Melanoma Research Alliance, n.d.). Stage II melanoma extends beyond the epidermis (very outer layer of skin) into the thicker dermis layer of the skin. It is thicker than Stage I melanoma and is slightly more likely to metastasize. There is still no evidence that Stage II melanoma has spread to the lymph tissues, lymph nodes, or body organs (Stage 2 Melanoma - Melanoma Research Alliance, n.d.). Stage III melanoma, also known as regional melanoma, has metastasized (spread) to nearby lymph nodes, lymph vessels, or skin. Lymph nodes are an important part of the lymphatic system, which is a vast network of tissues and organs that helps rid the body of waste, toxins, and other unwanted materials. The lymphatic system also helps support the immune system by transporting infectionfighting white blood cells throughout the body (Stage 2 Melanoma - Melanoma Research Alliance, n.d.). Stage IV melanoma has metastasized (spread) to other places throughout the body, such as the brain, lungs, liver, or gastrointestinal (GI) tract.

Melanoma may also have spread to distant points in the skin. Stage IV melanoma is considered distant metastatic melanoma (Stage 4 Melanoma - Melanoma Research Alliance, n.d.). Metastatic is the most advanced form of melanoma and is defined as cancer spreading to different parts of the body, including vital organs (Norman, 1952). Various mutations in genes have been found to contribute to melanoma, such as PROM1, CDK4, and PTGS2. While UV-induced melanoma survival rates are generally higher, UV-induced melanomas have a chance to become 
cancerous and transform into a metastatic melanoma. An estimated 60-70\% of cutaneous malignant melanomas are thought to be caused by ultraviolet (UV) radiation exposure (Freeman, 2018). Currently, no cure for metastatic melanoma exists. However, systemic therapies including cytotoxic chemotherapy, immunotherapy, and biochemotherapies are traditionally used (Bhatia et al., 2009). Moreover radiation therapy and medications such as aldesleukin, binimetinib, braftovi (Encorafenib) etc.(Drugs Approved for Melanoma - National Cancer Institute, n.d.). Several characteristics of cancer such as proliferation, cell cycle, and invasiveness will be described briefly here. Proliferation is an increase in the number of cells as a result of cell growth and cell division (Definition of Cell Proliferation - NCI Dictionary of Cancer Terms - National Cancer Institute, n.d.). Invasiveness is defined by tendation to spread especially to invade healthy tissue causing tumor growth. The need for additional individualized therapies for metastatic melanoma is highlighted in this review. Clustered Regularly Interspersed Short Palindromic Repeats- CRISPRassociated protein 9 CRISPR/Cas 9 system is a tool for genome editing in cells that can target specific beneficially as genetic variants for genome editing (Deshpande et al., 2016; Kato-Inui et al., 2018). Mechanism of CRISPR-Cas9 systems are at a low cost and have higher efficiency success rates than previous genome editing methods such as zinc finger nucleases (ZFN) or transcription activator-like effector nucleases (TALEN) in research (Li et al., 2020).

There are several advantages to CRISPR over ZNFs and TALENs. when it comes to comparing the systems. For example, target design simplicity, delivery of nucleic acids rather than large proteins, and the specificity for recognition conferred by the PAM sequence (Newman et al., 2008).CRISPR systems don't have to rely on protein formation, but only on the ribonucleotide complex. CRISPR Cas9 mechanisms of action include two genome modifications: knockouts through NHEJ(non-homologous end joining) and knockins through HDR(homologous recombination) (Non-Homologous End Joining, Homology-Directed Repair - Beckman Coulter, n.d.). Between the two, NHEJ is more predominantly used due to the following reasons. NHEJ break ends can be ligated without a homologous template, whereas HDR-breaks require a template to guide repair (Non-Homologous End Joining, Homology-Directed Repair - Beckman Coulter, n.d.). Moreover, NHEJ is more efficient than HDR due to HDR needing a more specific severed and intact donor strands of DNA. (Addgene, 2015). Gene editing using CRISPR-Cas9 offers the potential to treat melanoma through individualized targeting of genetic mutations. Efficiency, Specific modifications can be injected directly using RNAs encoding Cas9 protein and guideRNA (gRNA) into a selected injection location. In addition, CRISPR can introduce mutations to multiple genes by delivering multiple gRNAs, as Stadtmauer et al. demonstrated in their 2020 clinical trial the successful modification of PDCD1, TRAC, and TRAB genes in T cells.

In another clinical trial, CRISPR was used to treat a disorder caused by a mutation in CEP290 that leads to a non-functional protein. When this specific protein does not function rod cells in the retina die and light-gathering photoreceptors can't renew themselves, resulting in blindness. Cas 9 systems were used to make two cuts in the disrupted DNA. Results of the clinical trial were unclear as low doses of CRISPR can actually improve vision, however if the low doses are proven safe the patients will receive higher doses to improve vision (CRISPR Enters Its First Human Clinical Trials | Science News, n.d.). With results from these clinical trials, CRISPR can potentially be used ethically and successfully to improve patient prognosis. Gene editing using CRISPR-Cas9 offers the potential to treat melanoma through individualized targeting of genetic mutations. As cases rise and patient prognosis shortens, UVinduced melanoma and metastatic melanoma become a more pressing issue day by day. In several experimental studies, CRISPR- Cas9 systems have shown some insight into a potential cure for metastatic melanoma on an individual level. The knockout and knockdown of PTGS2, CD133, and CDK5 genes may be a potential solution for metastatic melanoma. The knockdown and knockout of these specific genes offer a potential solution for improving patient prognosis due to specific mutations within the gene causing melanoma. This review will compare gene editing that targets CD133, CDK5, and PTGS2 genes and offer insight into which approach will provide better prognosis for melanoma patients. In addition to the genetics underlying melanoma, factors such as gender and age will be considered as well as the potential uses for animal models in developing individualized therapies. 


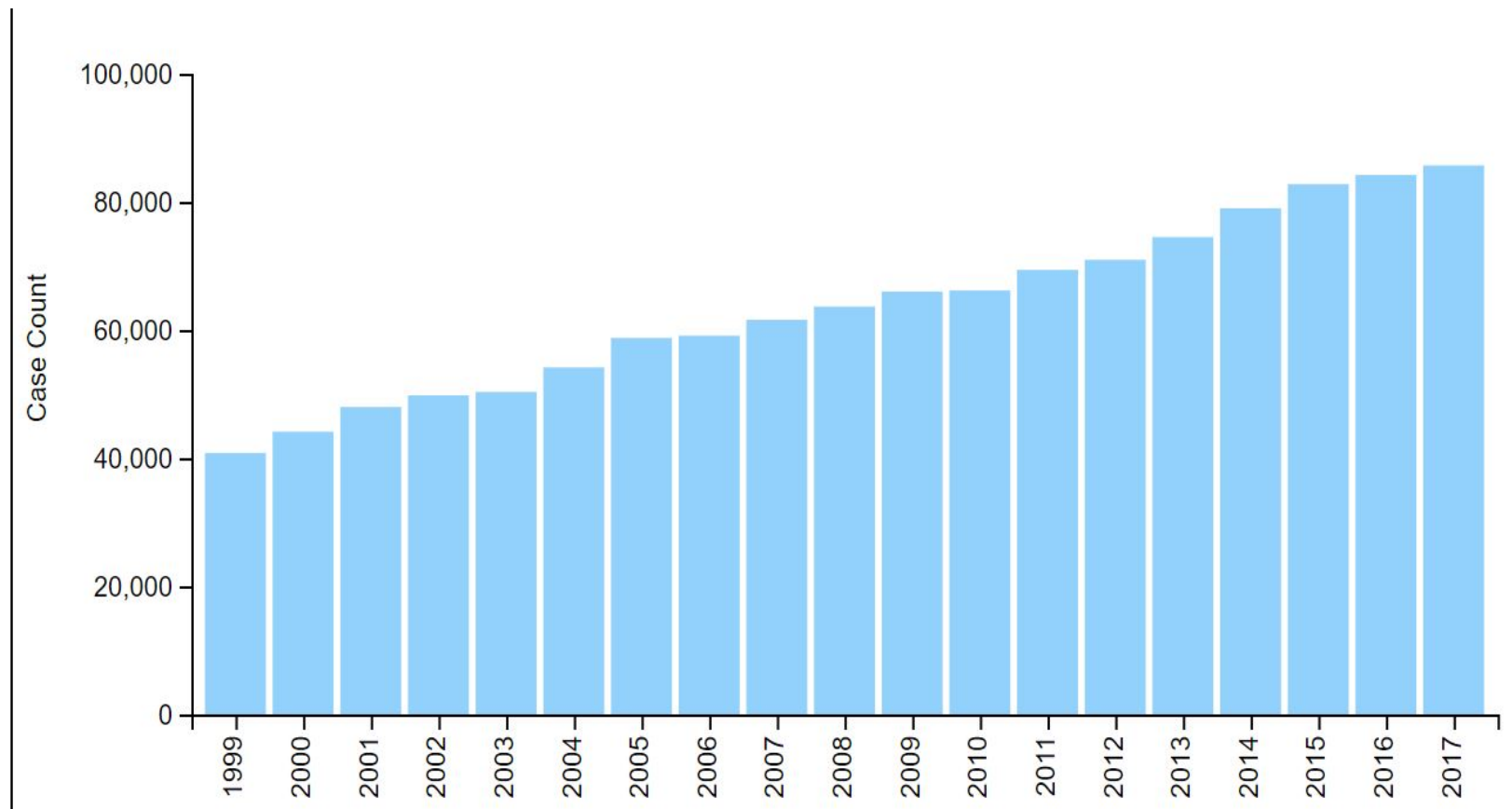

U.S. Cancer Statistics Working Group. U.S. Cancer Statistics Data Visualizations Tool, based on 2019 submission data (1999-2017): U.S. Department of Health and Human Services, Centers for Disease Control and Prevention and National Cancer Institute; www.cdc.gov/cancer/dataviz, released in June 2020.

The bar graph above represents the increase in melanoma cases in the US from 1999-2017. From this graph the general trend of melanoma increases year to year. This shows us that improvements in melanoma treatments are needed to inhibit further increases in melanoma cases.

\section{Current Treatments for Melanoma}

Patients with advanced melanoma receive numerous surgeries to resect tumor metastases. Currently, melanoma patients who present with metastatic disease and have undergone surgery, have a less than 30\% 5-year survival rate. State Source. In an article by Shailender Bhatua in 2009, 144 patients underwent surgical procedures to resect nonregional metastatic melanoma, where the general rates of survival were between 14 and 20 percent. Additionally, this paper found that the cohort of patients with more advanced metastatic melanoma had a survival rate of $10 \%$ after surgical resection. Patients were further stratified into those with complete or incomplete resection, where they have a $15 \%$ or $4 \% 5$-year survival rate. Surgical intervention may increase patient prognosis, but not significantly.

In addition to surgery, radiation therapy can be a front-line treatment against metastatic melanoma. UVinduced melanoma and metastatic melanoma are relatively radioresistant tumors. State Source. This causes most radiotherapies to be less effective in fighting against a tumor. Patient symptoms due to tumor metastasis can be improved with radiation therapy with radiation therapy. It is extremely useful in patients with central nervous system metastasis. Source. Another treatment for melanoma is systematic therapeutics. Systemic therapy refers to any type of cancer treatment that targets the entire body. Usually, consisting of substances passed through the bloodstream, to reach and affect cells all around the body (Systemic Therapy__Moffitt, n.d.). Lastly, systemic therapy is most used for patients with stage IV metastatic melanoma. Some examples of therapies include cytotoxic chemotherapy, immunotherapy, and bio chemotherapy. (Bhatia et al., 2009). Chemotherapy circulates through the bloodstream to destroy cancerous cells in multiple locations. Most of the time, chemotherapy is used to treat cancers that spread beyond the point of origination (Systemic Therapy_Moffitt, n.d.). While treatments for melanoma are effective, many patients still receive shorter prognosis. To lengthen patient prognosis, these treatments will need to be improved. 


\section{PROM 1}

PROM1, otherwise known as CD133, encodes for a Penta span transmembrane glycoprotein. This protein is usually found in adult stem cells by maintaining stem cell properties and suppressing differentiation. PROM 1 is commonly expressed in cancer stem cells that are isolated from various cancers including melanoma. (Saha et al., 2020). Several patients today are affected by PROM1 mutations, these mutations can be associated with the following diseases: breast cancer, acute myeloid leukemia, brain tumors, and lung cancer. PROM 1 mutations are also affiliated with poor patient prognosis (Saha et al., 2020). In the following paper by Cynthia M. Simbulan-Rosenthal et al, she states that their group investigated the role of PROM 1 in the invasiveness of minimum inhibitory concentration(MIC) and if this was mediated by MMP(mitochondrial membrane potential) secreted from melanocytes. This experiment used the knockout of PROM 1 in BAK-P-T3 melanoma cells using lentiviral delivery of CRISPR Cas9. With the knockout of PROM1 in the BAK-P-T3 cells, there was a significant decrease in the secretion of MMP2 and MMP9. These two genes are usually mutated several times in common diseases such as osteolysis and aortic aneurysms. ${ }^{45}$ Thus, the invasiveness of PROM1- expressing MIC's, may be mediated by the activation of MMP2 and MMP9 (Simbulan-Rosenthal et al., 2019). In addition, PROM 1 was expressed using a Dox-induced expression of the gene which resulted in increased invasion and metastasis. The paper also concludes that the data presented indicate PROM1 in MMP expression, invasion, and metastasis, and further support the use of PROM1 as a target for therapeutic intervention. Similarly, to the previous article, Chiou-Yan Lai et al found that PROM 1 expression in mouse melanoma models correlates with tumor progression in vivo. The study found out that, using flow cytometry, expression of PROM 1 increased as metastatic melanoma developed. This indicates that PROM 1 is more prevalent in mature disease states. Thus, in conclusion, the authors provided strong evidence that PROM 1 is prevalent in the growth of melanoma tumors and metastasis. CRISPR Cas9 will be useful in the knocking out of PROM 1 to suppress melanoma growth and further metastasis. In order to do this, gRNA will determine where the knockout will occur, when the gRNA and Cas9 is expressed in cells the gRNA will direct Cas9 to bind to a target sequence and introduce a double stranded break causing the PROM 1 gene to be knocked out. Thus, PROM 1 knockout can be a potential candidate for improving melanoma patient prognosis (Simbulan-Rosenthal et al., 2019).

\section{PTGS2}

Along with genetic manipulation of PROM1, the PTGS2 gene can potentially be used in melanoma therapeutics. PTGS2 is a protein encoding gene. Some diseases including PTGS2 are gastric ulcers and bursitis (Weizmann Institute of Science, 2020). PTGS2 is frequently expressed in malignant melanomas and its expression significantly correlates with poor survival in patients (Ercolano et al., 2019). In an article published by Giuseppe Ercolano et al, the authors found that the knockout of PTGS2 can reduce cell proliferation and colony formation (Ercolano et al., 2019). Using B160F10 melanoma cell lines, the PTGS2 gene was knocked out and the effects were assessed. As a result, the cell proliferation and colony formation was significantly decreased showing a potential therapeutic to melanoma. On the topic of cell invasion, the knockout of the PTGS2 gene also shows promise in reducing cell invasion and metastasis, also in B160F10 cells. The results of the experiment showed lower levels of invasiveness in the control cells compared to the PTGS2 knockout affected cell. Moreover, the researchers also tested PTGS2 suppression in metastatic melanoma in mice. The experiment consisted of the knockdown of PTGS2 in vivo, with implantation of B16/PTGS2 $\Delta$ in the mice. Results showed that the tumor reduced by $30 \%$ and there was a clear difference in control and affected mice. In conclusion, the article states that the knockdown of PTGS2 shows remarkable effects on tumorigenic properties in melanoma cells such as cell proliferation, colony formation capacity, migration, and invasiveness. Along with the PROM1 gene, CRISPR Cas9 systems could be used to improve the success rate of melanoma treatment using gene deletion methods. 


\section{CDK4}

CDK4 is an important gene in the cell cycle progression. This gene has been associated with several different cancer types. Diseases associated with CDK4 include melanoma, malignant melanoma, lung adenocarcinoma, conventional glioblastoma multiforme, dedifferentiated liposarcoma, breast invasive ductal carcinoma, and glioblastoma (CDK4 Gene - GeneCards _ CDK4 Protein _ CDK4 Antibody, n.d.)

Finally, the reduction of CDK4 is another potential treatment for melanoma.In an article by Marcelo L. et al , the deficiency of CDK4 inhibits skin tumor development. Using mouse models, CRISPR Cas9 delivered using a lentivirus to knockdown CDK4, resulting in a deficiency in CDK4 expression during cell proliferation. Compared with wildtype mice, the CDK4 knockdown exhibited a reduced size of metastasis. While this knockdown may seem effective, there are major risks associated with the deficiency of CDK4. Keratinocyte proliferation is the overproduction of keratinocytes, this results in an expansion of the cell population. Knocking down CDK4 in melanoma cells may increase the risk of further expansion of the tumor. In research by Marcelo L. et al, with the knockdown of CDK4, the authors did not find significant proliferative increases. In another article by Karoline Kollman et al., claims that CDK4 affects growth and survival of melanoma cell lines. This experiment consisted of mouse models to represent Stage IV melanoma. These mice were given either vehicle control or CDK4 inhibitor PD0332991. This led to a significant reduction of tumor growth compared to the original control group (Kollmann et al., 2019). Within the same article, also knocked down CDK4 and CDK6 which lead to decreased amounts of proliferation. The researchers performed a stable knockdown of CDK4 using lentiviral transfection in shRNA expressing vectors in 518A2 and LNM1 cells. Results of the experiment lead the researchers to believe CDK4 was important in melanoma and potential uses can be acquired. Migration of melanoma ultimately leads to shorter prognosis in patients. In the previous article, the researchers investigated the migration of melanoma from the origination site. In order to the migration rates of the cells, the researchers left the melanocytes with down regulated CDK4. This inturn, slowed the migration processes of the melanocyte cells. Therefore, knockdown of CDK4 could be a potential gene therapeutic for melanoma, though the threshold of knockdown remains to be determined (Kollmann et al., 2019). CRISPR Cas9 can be used in the knockdown of CDK4 through lentiviral delivery. Similar to PROM1 gene CDK4 is to be knockout, however CDK4 is vitally more important to cell growth and cell formation. The full knockout of CDK4 is less viable than PROM 1. Therefore CDK4 knockdown is a potential solution to improving patient prognosis, with some restrictions on the full knockout of the gene.

\section{Ethical Standpoint}

Since some melanomas are inherited through hereditary means, religion might condone CRISPR as disturbing God's creation and altering the way you are formed. This must be considered as many patients believe in these ideologies. CRISPR is experimental, many complications due to editing the genome might arise, ethically speaking, patients may refuse to continue with CRISPR gene editing. Improvements in gene editing through the use of clinical trials will further instill confidence in this therapeutic approach. Further, germline versus somatic mutations are often distinguished from one another in the setting of gene editing therapies. Some may argue that hereditary, or germline, mutations should not be altered, as this would entail embryonic stem cell editing. As previously mentioned, many people believe that the human genome should not be altered as it is God's creation. Germline mutations are mutations that can be passed to future offspring, thus many may argue with the passing down of altered genes versus individualized or somatic mutations. Due to ethical setbacks, CRISPR may not be a viable option for some patients. However, it is important that the general public be informed to fully understand the risks and implications, as well as potential ethical setbacks. 


\section{Discussion}

UV induced melanoma also affects the sexes differently. Men are more likely to receive UV-induced melanoma and die due to metastasis. Researchers believe that a major cause may lie in men's skin. We know that men's skin differs from women's skin. Men have thicker skin with less fat beneath. A man's skin also contains more collagen and elastin, fibers that give the skin firmness and keep it tight. Research shows that these differences make men's skin more likely to be damaged by the sun's ultraviolet (UV) rays. (Melanoma Strikes Men Harder, n.d.)

CRISPR can be used to treat both UV-induced melanoma and metastatic melanoma through the targeting of specific genes, including PROM1, PTGS2, and CDK4. Previously mentioned, UV-induced melanoma can lead to damage genetically ultimately causing metastases. If this is the case, CRISPR can be implemented to potentially reduce patient prognosis of UV-induced melanomas. CDK4 cannot be fully knocked out as in doing so many other complications arise such as off-target effects. A knockdown may be performed by CRISPR Cas 9 systems to reach the threshold for disease treatment. Ethical considerations can improve on reducing potential risks associated with deletion of genes. It can be improved with further testing involving non-human test subjects such as other animal models. All in all, between the knockout and knockdown of PROM1, PTGS2, and CDK4, it is PTGS2 that shows potential success in future gene therapeutics due to outcomes in mouse models, lower invasiveness, suppression of tumor growth, and lower risks when knocked out. Through the use of CRISPR Cas9 systems in both UV-induced melanoma and metastatic melanoma patient prognosis can potentially be improved to support the increase in melanoma cases year by year.

\section{Acknowledgments}

I would like to express my very great appreciation to Nicole Guilz for her valuable and constructive suggestions during the planning and development of this research work. Her willingness to give her time so generously has been very much appreciated.

I would also like to thank the following organization for their contributions to this paper:

Horizon Academic Research Program

\section{References}

Ercolano, G., De Cicco, P., Rubino, V., Terrazzano, G., Ruggiero, G., Carriero, R., Kunderfranco, P., \& Ianaro, A. (2019). Knockdown of PTGS2 by CRISPR/CAS9 system designates a new potential gene target for melanoma treatment. Frontiers in Pharmacology, 10(December), 1-12. https://doi.org/10.3389/fphar.2019.01456

Chiou, S., Winters, I. P., Wang, J., Naranjo, S., Dudgeon, C., Tamburini, F. B., Brady, J. J., Yang, D., Grüner, B. M., Chuang, C., Caswell, D. R., Zeng, H., Chu, P., Kim, G. E., Carpizo, D. R., Kim, S. K., \& Winslow, M. M. (2015). 2015.9. 1576-1585. https://doi.org/10.1101/gad.264861.115.1576

Dorothy A. Shead, M. D. (2018). NCCN Clinical Practice Guidelines in Oncology (NCCN Guidelines®) Version 1. 2017. E NCCN Quick Guide.

Survival, F. N. (2013). Men Women Net Survival (\%). 2013.

Ovchinnikov, D. A., Korn, O., Virshup, I., Wells, C. A., \& Wolvetang, E. J. (2018). The Impact of APP on Alzheimer-like Pathogenesis and Gene Expression in Down Syndrome iPSC-Derived Neurons. Stem Cell Reports, 11(1), 32-42. https://doi.org/10.1016/j.stemcr.2018.05.004 
Mosqueira, D., Mannhardt, I., Bhagwan, J. R., Lis-Slimak, K., Katili, P., Scott, E., Hassan, M., Prondzynski, M., Harmer, S. C., Tinker, A., Smith, J. G. W., Carrier, L., Williams, P. M., Gaffney, D., Eschenhagen, T., Hansen, A., \& Denning, C. (2018). CRISPR/Cas9 editing in human pluripotent stemcell-cardiomyocytes highlights arrhythmias, hypocontractility, and energy depletion as potential therapeutic targets for hypertrophic cardiomyopathy. European Heart Journal, 39(43), 3879-3892. https://doi.org/10.1093/eurheartj/ehy249

Callahan, S. J., Tepan, S., Zhang, Y. M., Lindsay, H., Burger, A., Campbell, N. R., Kim, I. S., Hollmann, T. J., Studer, L., Mosimann, C., \& White, R. M. (2018). Cancer modeling by Transgene Electroporation in Adult Zebrafish (TEAZ). Disease Models \& Mechanisms, 11(9). https://doi.org/10.1242/dmm.034561

Maraveyas, A., Johnson, M. J., Xiao, Y. P., \& Noble, S. (2010). Malignant melanoma as a target malignancy for the study of the anti-metastatic properties of the heparins. Cancer and Metastasis Reviews, 29(4), 777-784. https://doi.org/10.1007/s10555-010-9263-y

Tafazoli, A., Behjati, F., Farhud, D. D., \& Abbaszadegan, M. R. (2019). Combination of genetics and nanotechnology for down syndrome modification: A potential hypothesis and review of the literature. Iranian Journal of Public Health, 48(3), 371-378. https://doi.org/10.18502/ijph.v48i3.878

American Cancer Society. (2009). Melanoma of the Skin Staging. 1.

Deng, H., Tan, S., Gao, X., Zou, C., Xu, C., Tu, K., Song, Q., Fan, F., Huang, W., \& Zhang, Z. (2020). Cdk5 knocking out mediated by CRISPR-Cas9 genome editing for PD-L1 attenuation and enhanced antitumor immunity. Acta Pharmaceutica Sinica B, 10(2), 358-373. https://doi.org/10.1016/j.apsb.2019.07.004

Kim, I. S., Heilmann, S., Kansler, E. R., Zhang, Y., Zimmer, M., Ratnakumar, K., Bowman, R. L., Simon-Vermot, T., Fennell, M., Garippa, R., Lu, L., Lee, W., Hollmann, T., Xavier, J. B., \& White, R. M. (2017). Microenvironment-derived factors driving metastatic plasticity in melanoma. Nature Communications, 8(May 2016), 1-11. https://doi.org/10.1038/ncomms 14343

Mirus Bio LLC. (2018). Genome Editing : Crispr / Cas9 Delivery Methods. Shalem, O., Sanjana, N. E., Hartenian, E., Shi, X., Scott, D. A., Heckl, D., Ebert, B. L., Root, D. E., \& Doench, J. G. (2014). Shalem et al., 2014. Science, 343(6166), 84-87. https://doi.org/10.1126/science.1247005.Genome-Scale

Huang, H. (2018). Matrix metalloproteinase-9 (MMP-9) as a cancer biomarker and MMP-9 biosensors: Recent advances. Sensors (Switzerland), 18(10), 5-7. https://doi.org/10.3390/s18103249

Lowe, M. M., Boothby, I., Clancy, S., Ahn, R. S., Liao, W., Nguyen, D. N., Schumann, K., Marson, A., Mahuron, K. M., Kingsbury, G. A., Liu, Z., Sandoval, P. M., Rodriguez, R. S., Pauli, M. L., Taravati, K., Arron, S. T., Neuhaus, I. M., Harris, H. W., Kim, E. A., ... Rosenblum, M. D. (2019). Regulatory T cells use arginase 2 to enhance their metabolic fitness in tissues. JCI Insight, 4(24). https://doi.org/10.1172/jci.insight.129756

Snyder, D., Wang, Y., \& Kaetzel, D. M. (2020). A rare subpopulation of melanoma cells with low expression of metastasis suppressor NME1 is highly metastatic in vivo. Scientific Reports, 10(1), 1971.

https://doi.org/10.1038/s41598-020-58996-3

Wei, J., Long, L., Zheng, W., Dhungana, Y., Lim, S. A., Wang, Y., Wang, Y., Qian, C., Xu, B., Huang, H., Yu, J., Doench, J. G., \& Geiger, T. L. (2020). HHS Public Access. 576(7787), 471-476. https://doi.org/10.1038/s41586019-1821-z.Targeting 
Takeda, H., Kataoka, S., Nakayama, M., Ali, M. A. E., Oshima, H., Yamamoto, D., Park, J. W., Takegami, Y., An, T., Jenkins, N. A., Copeland, N. G., \& Oshima, M. (2019). CRISPR-Cas9-mediated gene knockout in intestinal tumor organoids provides functional validation for colorectal cancer driver genes. Proceedings of the National Academy of Sciences of the United States of America, 116(31), 15635-15644. https://doi.org/10.1073/pnas.1904714116

Pros and cons of ZNFs, TALENs, and CRISPR_Cas. (n.d.).

Lewis, B. C., Klimstra, D. S., \& Varmus, H. E. (2003). The c-myc and PyMT oncogenes induce different tumor types in a somatic mouse model for pancreatic cancer. Genes and Development, 17(24), 3127-3138.

https://doi.org/10.1101/gad.1140403

Falkson, C. I. (1995). Treatment of metastatic malignant melanoma. Anti-Cancer Drugs, 6(6), 709-716. https://doi.org/10.1097/00001813-199512000-00001

Vermersch, E., Jouve, C., \& Hulot, J. S. (2020). CRISPR/Cas9 gene-editing strategies in cardiovascular cells. Cardiovascular Research, 116(5), 894-907. https://doi.org/10.1093/cvr/cvz250

Del Castillo Velasco-Herrera, M., van der Weyden, L., Nsengimana, J., Speak, A. O., Sjöberg, M. K., Bishop, D. T., Jönsson, G., Newton-Bishop, J., \& Adams, D. J. (2018). Comparative genomics reveals that loss of lunatic fringe (LFNG) promotes melanoma metastasis. Molecular Oncology, 12(2), 239-255. https://doi.org/10.1002/1878$\underline{0261.12161}$

Ideno, N., Yamaguchi, H., Okumura, T., Huang, J., Brun, M. J., Ho, M. L., Suh, J., Gupta, S., Maitra, A., \& Ghosh, B. (2019). A pipeline for rapidly generating genetically engineered mouse models of pancreatic cancer using in vivo CRISPR-Cas9-mediated somatic recombination. Laboratory Investigation, 99(8), 1233-1244.

https://doi.org/10.1038/s41374-018-0171-z

Lezcano, C., Jungbluth, A. A., Nehal, K. S., Hollmann, T. J., Busam, K. J., Sloan, M., Cancer, K., Sloan, M., \& Cancer, K. (2019). HHS Public Access. 42(11), 1456-1465.

https://doi.org/10.1097/PAS.0000000000001134.PRAME

Koh, H. K. (1996). Prevention and Early Detection Strategies for Melanoma and Skin Cancer. In Archives of Dermatology (Vol. 132, Issue 4, p. 436). https://doi.org/10.1001/archderm.1996.03890280098014

Rashid, O. M., \& Takabe, K. (2014). Does removal of the primary tumor in metastatic breast cancer improve survival? Journal of Women's Health, 23(2), 184-188. https://doi.org/10.1089/jwh.2013.4517

Seidler, B., Schmidt, A., Mayr, U., Nakhai, H., Schmid, R. M., Schneider, G., \& Saur, D. (2008). A Cre-loxP-based mouse model for conditional somatic gene expression and knockdown in vivo by using avian retroviral vectors. Proceedings of the National Academy of Sciences of the United States of America, 105(29), 10137-10142.

https://doi.org/10.1073/pnas.0800487105

Bloh, K. M., Bialk, P. A., Gopalakrishnapillai, A., Kolb, E. A., \& Kmiec, E. B. (2017). CRISPR/Cas9-Directed Reassignment of the GATA1 Initiation Codon in K562 Cells to Recapitulate AML in Down Syndrome. Molecular Therapy - Nucleic Acids, 7(June), 288-298. https://doi.org/10.1016/j.omtn.2017.04.009

Roewenstrunk, J., Di Vona, C., Chen, J., Borras, E., Dong, C., Arató, K., Sabidó, E., Huen, M. S. Y., \& de la Luna, S. (2019). A comprehensive proteomics-based interaction screen that links DYRK1A to RNF169 and to the DNA damage response. Scientific Reports, 9(1), 1-14. https://doi.org/10.1038/s41598-019-42445-X 
Bouche, G., Jezdic, S., Dummer, R., \& Jost, L. (2014). Melanoma: a guide for patients - Information based on ESMO Clinical Practice Guidelines. 1-21

Akagi, K., Symer, D. E., Mohler, P. J., Ma, J., \& Paul, M. L. (2018). Cardiac Function in Dystrophic Mice. Circ Res., 121(8), 923-929. https://doi.org/10.1161/CIRCRESAHA.117.310996.In

Wang, T., Wei, J. J., Sabatini, D. M., \& Lander, E. S. (2012). Genetic screens in human cells using the CRISPRCas9 system. BMJ Supportive and Palliative Care, 2(3), 256-263. https://doi.org/10.1136/bmjspcare-2011-000063 Albert, D. M., Niffenegger, A. S., \& Willson, J. K. (1992). Treatment of metastatic uveal melanoma: review and recommendations. Survey of Ophthalmology, 36(6), 429-438. https://doi.org/10.1016/s0039-6257(05)80024-4

Christodoulou, E., Rashid, M., Pacini, C., Alastair, D., Robertson, H., van Groningen, T., Teunisse, A. F. A. S., Iorio, F., Jochemsen, A. G., Adams, D. J., \& van Doorn, R. (2020). Analysis of CRISPR-Cas9 screens identify genetic dependencies in melanoma. Pigment Cell \& Melanoma Research. https://doi.org/10.1111/pcmr.12919

Fitzpatrick, T. B., Montgomery, H., \& Lerner, A. B. (1954). Pathogenesis of Generalized Dermal Pigmentation Secondary to Malignant Melanoma and Melanuria*. Journal of Investigative Dermatology, 22(3), $163-172$. https://doi.org/10.1038/jid.1954.22

Meyers, R. M., Bryan, J. G., Mcfarland, J. M., Weir, B. A., Ann, E., Xu, H., Dharia, N. V, Montgomery, P. G., Cowley, G. S., Pantel, S., Goodale, A., Lee, Y., Ali, L. D., Jiang, G., Lubonja, R., Harrington, W. F., Strickland, M., Wu, T., Hawes, D. C., ... David, E. (2018). HHS Public Access. 49(12), 1779-1784.

https://doi.org/10.1038/ng.3984.Computational

Peter K. Smith Jess Mahdavi Manuel Carvalho Sonja Fisher Shanette Russell Neil Tippett. (2008). J @ Onlinelibrary.Wiley.Com. In Journal of Child Psychology and Psychiatry (Vol. 49, Issue 4, p. [1] Peter K. Smith Jess Mahdavi Manuel Carvalho So). https://doi.org/10.1080/0144039X.2013.791174

Ekman, F. K., Ojala, D. S., Adil, M. M., Lopez, P. A., Schaffer, D. V., \& Gaj, T. (2019). CRISPR-Cas9-Mediated Genome Editing Increases Lifespan and Improves Motor Deficits in a Huntington's Disease Mouse Model. Molecular Therapy - Nucleic Acids, 17(September), 829-839. https://doi.org/10.1016/j.omtn.2019.07.009

Shin, J. W., Kim, K. H., Chao, M. J., Atwal, R. S., Gillis, T., MacDonald, M. E., Gusella, J. F., \& Lee, J. M. (2016). Permanent inactivation of Huntington's disease mutation by personalized allele-specific CRISPR/Cas9. Human Molecular Genetics, 25(20), 4566-4576. https://doi.org/10.1093/hmg/ddw286

Reed, K. B., Cook-Norris, R. H., \& Brewer, J. D. (2012). The cutaneous manifestations of metastatic malignant melanoma. International Journal of Dermatology, 51(3), 243-249. https://doi.org/10.1111/j.1365-4632.2011.05245.x

Non-Homologous End Joining, Homology-Directed Repair - Beckman Coulter. (n.d.). https://www.beckman.com/support/faq/research/nhej-hdr-difference

CRISPR enters its first human clinical trials | Science News. (n.d.). https://www.sciencenews.org/article/crispr-geneeditor-first-human-clinical-trials

Addgene. (2015). CRISPR 101: Non-Homologous End Joining. In Addgenes's blog. 
Simbulan-Rosenthal, C. M., Gaur, A., Zhou, H., Abdussamad, M., Qin, Q., Dougherty, R., Aljehane, L., Kuo, L. W., Vakili, S., Karna, K., Clark, H., McCarron, E., \& Rosenthal, D. S. (2019). CD133 Is Associated with Increased Melanoma Cell Survival after Multikinase Inhibition. Journal of Oncology, 2019. https://doi.org/10.1155/2019/6486173

Weizmann Institute of Science. (2020). PTGS2 Gene - GeneCards | PGH2 Protein | PGH2 Antibody. https://www.genecards.org/cgi-bin/carddisp.pl?gene=PTGS2\&keywords=ptgs2

Kollmann, K., Briand, C., Bellutti, F., Schicher, N., Blunder, S., Zojer, M., \& Hoeller, C. (2019). The interplay of CDK4 and CDK6 in melanoma. Oncotarget, 10(14), 1346-1359. https://doi.org/10.18632/oncotarget.26515

Melanoma strikes men harder. (n.d.). https://www.aad.org/public/diseases/skin-cancer/types/common/melanoma/men-50

CDK4 Gene - GeneCards _CDK4 Protein _CDK4 Antibody. (n.d.).

USCSChart. (n.d.).

Stage 2 Melanoma - Melanoma Research Alliance. (n.d.).

Definition of cell proliferation - NCI Dictionary of Cancer Terms - National Cancer Institute. (n.d.). https://www.cancer.gov/publications/dictionaries/cancer-terms?cdrid $=46479$

Kato-Inui, T., Takahashi, G., Hsu, S., \& Miyaoka, Y. (2018). Clustered regularly interspaced short palindromic repeats (CRISPR)/CRISPR-associated protein 9 with improved proof-reading enhances homology-directed repair. $\mathrm{Nu}$ cleic Acids Research, 46(9), 4677-4688. https://doi.org/10.1093/nar/gky264

Drugs Approved for Melanoma - National Cancer Institute. (n.d.). https://www.cancer.gov/about-cancer/treatment/drugs/melanoma Systemic Therapy_Moffitt. (n.d.).

Stage 3 Melanoma. (n.d.). http://www.bad.org.uk/shared/get-file.ashx?id=103\&itemtype=document

Li, H., Yang, Y., Hong, W., Huang, M., Wu, M., \& Zhao, X. (2020). Applications of genome editing technology in the targeted therapy of human diseases: mechanisms, advances and prospects. In Signal Transduction and Targeted Therapy (Vol. 5, Issue 1). https://doi.org/10.1038/s41392-019-0089-y

2007, N., Gaj, T., Gersbach, C. A., \& Barbas, C. F. (2008). ZFN, TALEN and CRISPR/Cas-based methods for genome engineering NIH Public Access. Bone, 23(1), 1-7. https://doi.org/10.1016/j.tibtech.2013.04.004.ZFN

Stage 4 Melanoma - Melanoma Research Alliance. (n.d.).

Definition of COLPOSCOPY hemangioma - NCI Dictionary of Cancer Terms - National Cancer Institute NCI Dictionary of Cancer Terms (p. 2019). (2019).

Stage 1 Melanoma - Melanoma Research Alliance. (n.d.).

Deshpande, K., Vyas, A., Balakrishnan, A., \& Vyas, D. (2016). Clustered Regularly Interspaced Short Palindromic Repeats/Cas9 Genetic Engineering: Robotic Genetic Surgery. American Journal of Robotic Surgery, 2(1), 49-52. https://doi.org/10.1166/ajrs.2015.1023

Norman, A. (1952). On the origin of cancer foci. Cancer, 5(3), 581-582. https://doi.org/10.1002/10970142(195205)5:3<581::AID-CNCR2820050319>3.0.CO;2-Q 
Rodriguez-Puebla, M. L., Miliani de Marval, P. L., LaCava, M., Moons, D. S., Kiyokawa, H., \& Conti, C. J. (2002). Cdk4 Deficiency Inhibits Skin Tumor Development But Does Not Affect Normal Keratinocyte Proliferation. American Journal of Pathology, 161(2), 405-411. https://doi.org/10.1016/S0002-9440(10)64196-X

Saha, S. K., Islam, S. M. R., Kwak, K. S., Rahman, M. S., \& Cho, S. G. (2020). PROM1 and PROM2 expression differentially modulates clinical prognosis of cancer: a multiomics analysis. Cancer Gene Therapy, 27(3-4), 147167. https://doi.org/10.1038/s41417-019-0109-7

Bhatia, S., Tykodi, S. S., \& Thompson, J. A. (2009). Treatment of metastatic melanoma: An overview. Oncology, 23(6), 488-496.

Sandru, A., Voinea, S., Panaitescu, E., \& Blidaru, A. (2014). Survival rates of patients with metastatic malignant melanoma. Journal of Medicine and Life, 7(4), 572-576.

Freeman. (2018). 乳鼠心肌提取 HHS Public Access. Physiology \& Behavior, 176(1), 139-148.

https://doi.org/10.1111/phpp.12329.Mechanisms

Simbulan-Rosenthal, C. M., Dougherty, R., Vakili, S., Ferraro, A. M., Kuo, L. W., Alobaidi, R., Aljehane, L., Gaur, A., Sykora, P., Glasgow, E., Agarwal, S., \& Rosenthal, D. S. (2019). CRISPR-Cas9 knockdown and induced expression of CD133 reveal essential roles in melanoma invasion and metastasis. Cancers, 11(10), 1-23.

https://doi.org/10.3390/cancers 11101490

Zeng, H., Jorapur, A., Shain, A. H., Lang, U. E., Zhang, Y., Mcneal, A. S., Botton, T., Lin, J., Bastian, I. N., Yu, R., North, J. P., Pincus, L., Ruben, S., Joseph, N. M., Yeh, I., Bastian, B. C., \& Judson, R. L. (2019). activation. 34(1), 56-68. https://doi.org/10.1016/j.ccell.2018.05.014.Bi-allelic

Doudna, J. A., \& Charpentier, E. (2014). The new frontier of genome engineering with CRISPR-Cas9. Science, 346(6213). https://doi.org/10.1126/science.1258096 